\title{
ON THE SOLVABILITY OF GROUPS OF CENTRAL TYPE ${ }^{1}$
}

\section{JAY YELLEN}

ABSTRACT. Let $G$ be a finite group with center $Z$ and irreducible complex character $x$ so that $\chi(1)^{2}=[G: Z]$. If the 2-Sylow subgroup of $G / Z$ has order 16 or less then $G$ is solvable.

Introduction. Let $G$ be a finite group with center $Z$. If $G$ has an irreducible complex character $\chi$ with $\chi(1)^{2}=[G: Z]$, then $G$ is called a group of central type [4]. It was conjectured in [8] that groups of central type are solvable and several authors have given partial results in this direction [2][4], [6], [9], and [12]. For example, in [4] it is proved that if $G$ is a group of central type and if for any prime $p, p^{m} \mid[G: Z]$ implies $m \leq 2$ then $G$ is solvable. In. [6], the integer 2 in this result was replaced by 4 . Here we show that if $2^{m} \mid[G: Z]$ implies $m \leq 4$ and $G$ is of central type, then $G$ is solvable. The proof employs the well-known characterizations of simple groups with small 2-Sylow subgroups and information on possible homomorphic images of groups of central type given in [6]. All unexplained notation and terminology is as in [7].

Lemma 1. Let $G$ be a finite group having a nonabelian composition factor $S$ appearing exactly $n$ times. Then there exist a homomorphic image $X$ of $G$ and an integer $m$ such that $1 \leq m \leq n$ and $S_{1} \times \ldots \times S_{m} \leq X \leq$ $\operatorname{Aut}\left(S_{1} \times \ldots \times S_{m}\right)$ where $S_{i} \simeq S$ for all $i=1,2, \ldots, m$.

Proof. Use induction on $|G|$. Let $T$ be a minimal nontrivial normal subgroup of $G$. If $G$ is simple then $G=S$ and $S \leq G \leq \operatorname{Aut}(S)$. So we may assume $\{1\}<T<G$. We have $T=T_{1} \times \ldots \times T_{k}$ where the $T_{i}$ are isomorphic simple groups, $i=1,2, \ldots, k$.

Case 1. If $T_{i} \not S$ for all $i=1,2, \ldots, k$, then consider $G_{1}=G / T$. By the inductive hypothesis there exists a homomorphic image $X$ of $G_{1}$ and an integer $m$ such that $1 \leq m \leq n$ and $S_{1} \times \ldots \times S_{m} \leq X \leq \operatorname{Aut}\left(S_{1} \times \ldots \times S_{m}\right)$, where $S_{i} \simeq S$. This is the desired homomorphic image $X$ of $G$.

Case 2. If $T_{i} \simeq S$ for all $i=1,2, \ldots, k$, then $T \simeq S_{1} \times \ldots \times S_{k}, k \leq$ $n$ and $S_{i} \simeq S$. Since $T \Delta G, C_{G}(T) \Delta G$ and we have $G / C_{G}(T) \leq \operatorname{Aut}(T)$. Since $T$ is minimal normal, $C_{G}(T) \cap T=\{1\}$ and thus

Received by the editors July 31, 1974.

AMS (MOS) subject classifications (1970). Primary 20C15, 20D10; Secondary 20D05, $20 \mathrm{D} 45$.

Key words and phrases. Finite group, irreducible complex character, solvable group, simple group.

1 This work forms part of the author's Doctoral Dissertation at Colorado State University written under the direction of F. R. DeMeyer.

Copyright $\odot$ 1975, American Mathematical Society 


$$
T \simeq T \cdot C_{G}(T) / C_{G}(T) \leq G / C_{G}(T) \leq \operatorname{Aut}(T) .
$$

Lemma 2. Let $G$ be a group of central type with a normal subgroup $K$ such that $G / K$ has a cyclic p-Sylow subgroup. Then $G / K$ bas a p-complement. Furthermore, if $G / K$ is also simple then the prime $p$ is unique.

Proof. [6, 2.4(a) and its proof].

Lemma 3. Suppose $S \leq X \leq \operatorname{Aut}(S)$, where $S=P S L(2, q), q$ odd and $q>$ 3 , or $S=A_{7}$. Then $X$ is not the homomorphic image of a group of central type.

Proof. [6, 4.3 and the proof of 4.4].

The following number theoretic results are needed.

Lemma 4 (Zigmundy, 1896). Let $a$ and $n$ be integers both greater than one. Then there exists a prime divisor $p$ of $a^{n}-1$ such that $p \backslash a^{m}-1$ for all $m$ satisfying $1 \leq m<n$, except for the following cases:

(a) $n=2$ and $a$ is a Mersenne number.

(b) $n=6$ and $a=2$.

Prool. [1].

Lemma 5. Let $a$ and $k$ be positive integers. Then there exists an odd prime $p$ such that $p \mid a^{2 k+1}-1$ and $p \nmid 2 k+1$.

Proof. By Lemma 4, there is a prime divisor $p$ of $a^{2 k+1}-1$ such that $p \nmid a^{m}-1$ for all $1 \leq m<2 k+1 . p \mid a^{p-1}-1$ by Fermat's little theorem. Hence $p-1 \geq 2 k+1$, so $p>2 k+1$. Therefore $p$ is odd and $p \not 2 k+1$.

Theorem 1. Let $G$ be a group of central type with center $Z$. If the 2Sylow subgroup of $G / Z$ has order 16 or less, then $G$ is solvable.

Proof. If the 2-Sylow subgroup of $G / Z$ is trivial then $G / Z$ has odd order and hence is solvable. Since $[G: Z]$ is a square, the 2-Sylow subgroup of $G / Z$ has order 4 or 16 . If $G / Z$ is nonsolvable, then it must have at least one nonabelian composition factor. There are several possibilities and these possibilities are considered by cases.

Case 1. Assume a 2-Sylow subgroup $P$ of $G / Z$ has order 4. Then $P=$ $C_{2} \times C_{2}$ by [4, Theorem 2 and Lemma 2]. If $G / Z$ has a nonabelian composition factor $S$ then its 2-Sylow subgroup must be $C_{2} \times C_{2}$, since no nonabelian simple group can have $C_{2}$ as its 2-Sylow subgroup. Also $S$ appears exactly once since the 2-Sylow subgroup of $G / Z$ has order 4. Applying Lemma $1, S \leq X \leq \operatorname{Aut}(S)$ where $X$ is a homomorphic image of $G / Z$. Since $S$ is a simple group whose 2-Sylow subgroup is $C_{2} \times C_{2}, S=P S L\left(2, p^{n}\right)$ where $p^{n}>3$ and $p$ is an odd prime [5, 4.2.3]. So $\operatorname{PSL}\left(2, p^{n}\right) \leq X \leq \operatorname{Aut}\left(P S L\left(2, p^{n}\right)\right)$. By Lemma $3, X$ is not a homomorphic image of a group of central type and this contradiction completes Case 1 . 
Case 2. Assume a 2-Sylow subgroup of $G / Z$ has order 16 .

Subcase 1. Assume $G / Z$ has a nonabelian composition factor $S$ having a 2-Sylow subgroup $P$ of order 16 . The only groups of order 16 which can occur as 2-Sylow subgroups of simple groups are the elementary abelian, the dihedral, and the semidihedral groups [5]. $P$ is of central type [4, Theorem $2]$ and so by $[6,3.2]$, the dihedral and semidihedral groups are eliminated since they have cyclic self-centralizing subgroups. Hence $P$ is elementary abelian of order 16. By [5, 4.2.3], $S=P S L(2, q), q \equiv \pm 3(\bmod 8)$ or $S=$ $S L_{2}(16)$. The first possibility has already been ruled out in Case 1 , so suppose $S=S L_{2}(16)$. By Lemma 1 ,

$$
S L_{2}(16) \leq X \leq \operatorname{Aut}\left(S L_{2}(16)\right)=\Gamma L(2,16) .
$$

We also have $\left|S L_{2}(16)\right|=15 \cdot 16 \cdot 17$. Consider the cyclic 5-Sylow subgroup $Q$ of $S L_{2}(16)$. If $S L_{2}(16)$ had a 5-complement, then $S L_{2}$ (16) would act faithfully on the 5 cosets of the complement, and hence $S L_{2}(16)$ would be a subgroup of $S_{5}$, which is impossible. Thus $S L_{2}(16)$ has no 5-complement. Now $\left[X: S L_{2}(16)\right]$ divides $\left[\Gamma L(2,16): S L_{2}(16)\right]=4$, so $5 X\left[X: S L_{2}(16)\right]$, therefore $Q$ is the 5 Sylow subgroup of $X$. Suppose $Z \leq K \leq H \leq G$, where $K, H \triangle G, H / K \simeq$ $S L_{2}(16)$ and $G / K \simeq X$. By Lemma $2, G / K$ has a 5-complement, say $T / K$. The second isomorphism theorem and $5 \not G: H]$ imply that $H / K \cap T / K$ is a 5 -complement for $H / K$, which is a contradiction.

Subcase 2. Assume $G / Z$ has a nonabelian composition factor $S$ having a 2-Sylow subgroup of order 8 . There are five possibilities [5]:

(a) $S=P S L(2, q), q$ odd, $q>3$.

(b) $S=A_{7}$.

(c) $S=J(11)$, the simple group discovered by Janko.

(d) $S=S L_{2}(8)$.

(e) $S$ is a group of Ree type.

We rule out $\operatorname{PSL}(2, q)$ and $A_{7}$ by Lemmas 1 and 3 . Suppose $S=J(11)$. Outer $(S)=1[5]$ and $S \leq X \leq \operatorname{Aut}(S)$ by Lemma 1 ; hence $X=S$ where $X$ is a homomorphic image of $G / Z$. But $|J(11)|=2^{3} \cdot 3 \cdot 5 \cdot 7 \cdot 11 \cdot 19$, which contradicts Lemma 2.

Next suppose $S=S L_{2}(8) .|S|=7 \cdot 8 \cdot 9$. By Lemma $1, S \leq X \leq \operatorname{Aut}(S)$, where $[\operatorname{Aut}(S): S]=3$. Thus $7 \chi[X: S]$ and hence a cyclic 7-Sylow subgroup of $S$ is also a 7-Sylow subgroup of $X . X$ has a 7-complement by Lemma 2, and so $S$ also has a 7 -complement. $S$ acts faithfully on the 7 cosets of the complement, so we have $S \leq S_{7}$. This is impossible as $S L_{2}(8)$ has a cyclic subgroup of order 9 .

The remaining possibility is a group $S$ of Ree type. Then $|S|=$ $q^{3}(q-1)\left(q^{3}+1\right)$, where $q=3^{2 k+1}, k \geq 1$. $S$ has a cyclic subgroup $W$ of order $q-1[13$, p. 63$]$. By Lemma 5 , there exists an odd prime $p$ dividing 
$|W|$ but not dividing $2 k+1$. There is a cyclic $p$-Sylow subgroup $P$ of $W$, and since $\left(q-1, q^{3}+1\right)=2, P$ is also a $p$-Sylow subgroup of $S$. By Lemma $1, S \leq X \leq \operatorname{Aut}(S)$, where $X$ is a homomorphic image of $G / Z$. $[\operatorname{Aut}(S): S]=$ $2 k+1[10,9.1]$. Thus $p \nmid[X: S]$ and hence $P$ is a cyclic $p$-Sylow subgroup of $X . X$ has a $p$-complement by Lemma 2 , and hence $S$ has a $p$-complement. $S$ acts faithfully on the $|P|$ cosets of the complement and $|P|$ divides $|W|=$ $q-1$. Therefore $S$ has a permutation representation of degree less than or equal to $q-1$, with the 1-character occurring only once. But in the character table of a group of Ree type [13, p. 87] there are no irreducible characters of degree less than or equal to $q-1$.

Subcase 3. Assume $G / Z$ has a nonabelian composition factor $S$ having 2-Sylow subgroup of order 4. This has been ruled out in Case 1.

Subcase 4. Assume $G / Z$ has two nonabelian composition factors; $S_{1}$ and $S_{2}$, each having a 2-Sylow subgroup of order 4 . Repeating the argument in Case 1 we see that $S_{1} \simeq S_{2} \simeq \operatorname{PSL}\left(2, p^{n}\right)$ where $p^{n}>3$ and $p$ is an odd prime. $S_{1} \leq X \leq \operatorname{Aut}\left(S_{1}\right)$ is not possible by Case 1 , so by Lemma $1, S_{1} \times S_{2}$ $\leq X \leq \operatorname{Aut}\left(S_{1} \times S_{2}\right)$, where $X$ is a homomorphic image of $G / Z$. Let $\sigma \epsilon$ $\operatorname{Aut}\left(S_{1} \times S_{2}\right)$. Using a Krull-Schmidt argument [11, 4.6.3], we can regard $\sigma$ as a permutation on two letters, and the kernel of this action is $\operatorname{Aut}\left(S_{1}\right) \times$ $\operatorname{Aut}\left(S_{2}\right)$. Therefore

$$
\operatorname{Aut}\left(S_{1} \times S_{2}\right) / \operatorname{Aut}\left(S_{1}\right) \times \operatorname{Aut}\left(S_{2}\right) \simeq C_{2} \text {. }
$$

We consider two possibilities. First suppose $S_{1} \times S_{2} \leq X \leq \operatorname{Aut}\left(S_{1}\right) \times \operatorname{Aut}\left(S_{2}\right)$. By projecting onto $\operatorname{Aut}\left(S_{1}\right)$ we get

and so

$$
\left(S_{1} \times S_{2}\right) \operatorname{Aut}\left(S_{2}\right) / \operatorname{Aut}\left(S_{2}\right) \leq X \operatorname{Aut}\left(S_{2}\right) / \operatorname{Aut}\left(S_{2}\right) \leq \operatorname{Aut}\left(S_{1}\right)
$$

$$
\left(S_{1} \times S_{2}\right) /\left(S_{1} \times S_{2}\right) \cap \operatorname{Aut}\left(S_{2}\right) \leq X / \operatorname{Aut}\left(S_{2}\right) \cap X \leq \operatorname{Aut}\left(S_{1}\right) .
$$

But $\left(S_{1} \times S_{2}\right) \cap \operatorname{Aut}\left(S_{2}\right)=S_{2}$, thus $S_{1} \leq Y \leq \operatorname{Aut}\left(S_{1}\right)$ where $Y$ is a homomorphic image of $G / Z$. Now apply Lemma 3 to get the desired contradiction. Since $\left[\operatorname{Aut}\left(S_{1} \times S_{2}\right): \operatorname{Aut}\left(S_{1}\right) \times \operatorname{Aut}\left(S_{2}\right)\right]=2$. the remaining possibility is when $X=\operatorname{Aut}\left(S_{1} \times S_{2}\right)$. This is ruled out because the 2-Sylow subgroup of $X$ would have order greater than 16 . This completes the proof.

The author wishes to thank F. R. DeMeyer and R. Liebler for their invaluable advice during the preparation of this paper. R. Liebler's suggestion of Lemma 1 was especially helpful. The author also wishes to thank W. Brandal for his careful proofreading and helpful comments regarding the style and structure of the paper.

\section{BIBLIOGRAPHY}

1. G. D. Birkhoff and H. S. Vandiver, "On the integral divisors of $a^{n}-b^{n}, "$ in George David Birkhoff: Collected mathematical papers. Vol. 3, Amer. Math. Soc., Providence, R. I., 1950, pp. 145-152. 
2. F. R. DeMeyer, Groups with an irreducible character of large degree are solvable, Proc. Amer. Math. Soc. 25 (1970), 615-617. MR 43 \#368.

3. - Irreducible characters and solvability of finite groups, Pacific J. Math. 41 (1972), 347-353. MR 48 \#402.

4. F. R. DeMeyer and G. J. Janusz, Finite groups with an irreducible representation of large degree, Math. Z. 108 (1969), 145-153. MR 38 \#5910.

5. W. Feit, The current situation on the theory of finite simple groups, Proc. Internat. Congress Math. (Nice, 1970), vol. 1, Gauthier-Villars, Paris, 1971, pp. 5593.

6. S. M. Gagola, Characters fully ramified over a normal subgroup, University of Wisconsin (preprint).

7. D. Gorenstein, Finite groups, Harper \& Row, New York, 1968. MR 38 \#229.

8. H. Matsumoto and N. Iwahori, Several remarks on projective representations of finite groups, J. Fac. Sci. Univ. Tokyo Sect. I 10 (1964), 129-146. MR 31 \#4841.

9. H. Pahlings, Gruppen mit irreduziblen Darstellungen hohen Grades, Mitt. Math. Sem. Giessen 85 (1970), 27-44. MR 41 \#8537.

10. R. Ree, A family of simple groups associated with the simple Lie algebra of type $\left(G_{2}\right)$, Amer. J. Math. 83 (1961), 432-462. MR $25 \# 2123$.

11. W. R. Scott, Group theory, Prentice-Hall, Englewood Cliffs, N. J., 1964. MR $29 \# 4785$.

12. K. Timmer, Subgroups of groups of central type, Trans. Amer. Math. Soc. 189 (1974), 133-161.

13. H. N. Ward, On Ree's series of simple groups, Trans. Amer. Math. Soc. 121 (1966), 62-89. MR $33 \# 5752$.

DEPARTMENT OF MATHEMATICS, COLORADO STATE UNIVERSITY, FORT COLLINS, COLORADO 80521

Current address: Department of Mathematics, Allegheny College, Meadville, Pennsylvania 16335 